\title{
The Even-Odd Rule on Single Covalent-Bonded Structural Formulas as a Modification of Classical Structural Formulas of Multiple-Bonded Ions and Molecules
}

\author{
Geoffroy Auvert \\ CEA-Leti, Grenoble, France \\ Email: Geoffroy.auvert@grenoble-inp.org
}

Received 18 September 2014; revised 15 October 2014; accepted 10 November 2014

Copyright (C) 2014 by author and Scientific Research Publishing Inc.

This work is licensed under the Creative Commons Attribution International License (CC BY). http://creativecommons.org/licenses/by/4.0/

(c) †) Open Access

\begin{abstract}
In organic chemistry, as defined by Abegg, Kossel, Lewis and Langmuir, compounds are normally represented using structural formulas called Lewis structures. In these structures, the octet rule is used to define the number of covalent bonds that each atom forms with its neighbors and multiple bonds are frequent. Lewis' octet rule has unfortunately shown limitations very early when applied to non-organic compounds: most of them remain incompatible with the "rule of eight" and location of charges is uncertain. In an attempt to unify structural formulas of octet and non-octet molecules or single-charge ions, an even-odd rule was recently proposed, together with a procedure to locate charge precisely. This even-odd rule has introduced a charge-dependent effective-valence number calculated for each atom. With this number and the number of covalent bonds of each element, two even numbers are calculated. These numbers are both used to understand and draw structural formulas of single-covalent-bonded compounds. In the present paper, a procedure is proposed to adjust structural formulas of compounds that are commonly represented with multiple bonds. In order to keep them compatible with the even-odd rule, they will be represented using only single covalent bonds. The procedure will then describe the consequences of bond simplification on charges locations. The newly obtained representations are compared to their conventional structural formulas, i.e. single-bond representation vs. multiple-bond structures. Throughout the comparison process, charges are precisely located and assigned to specific atoms. After discussion of particular cases of compounds, the paper finally concludes that a rule limiting representations of multiple covalent bonds to single covalent bonds, seems to be suitable for numerous known compounds.
\end{abstract}




\section{Keywords}

\section{Multiple Bond, Single Bond, Covalent Bond, Molecule, Ion, Even-Odd, Rule, Structural Formula}

\section{Introduction}

In classical 2D structural formulas of ions and molecules, single bonds are represented by drawing one line between next neighbor atoms, like in di-hydrogen [1]. Other molecules are represented with multiple bonds, i.e. several lines between connected atoms: For instance in di-nitrogen where nitrogen atoms are connected with three lines [2]. These different types of bonds have been in combination with the octet rule to represent molecules for about one century [3]-[5]. Lewis' octet rule, at first successful in representing organic molecules, has later shown limitations, mainly for non-organic molecules [6] [7]. In an attempt to address both octet and non-octet molecules, a new rule, named the "even-odd" rule, was recently proposed and shown to apply to molecules like Li2 [8]. This rule was subsequently checked against ions involving only single-bonded interconnections between neighbors [9]. The even-odd rule seems to be generally capable of representing many single-bonded compounds and well-known octet or non-octet compounds [8] [9].

The aim of the present paper is to widen the scope of the even-odd rule by addressing multiple-bonded compounds. In the process, it proposes an adjustment to the representation of multiple-bonded ions and molecules. The proposed modification does not affect the position of atoms in ions or molecules, but only impacts the representation of interconnections between atoms.

This paper thus begins by describing a method to convert multiple bonds into single bonds in ions and molecules. Then a definition of the even-odd rule is given. Many types of multiple-bonded compounds are then redrawn by applying the conversion method and the even-odd rule. This includes octet and non-octet compounds in order to illustrate the versatility of the even-odd rule. At last, a discussion is proposed to point out important cases like the unambiguous determination of charge position in both charge-neutralized molecules and multiple-charged ions.

\section{Redefining Multiple Bonds as Single Bonds}

In this paper, a modification in the drawing of multiple-bonded connections between atoms in molecules or ions is proposed as follows:

- A single covalent bond between two first neighbors classically drawn with one line, is not modified.

- A classical representation of a double-bond is replaced by a single bond and by adding one positive charge on one connected atoms and a negative charge to the other one.

- A triple bond is replaced by a single covalent bond without any other impact.

- A quadruple bond is transformed into a single bond using the same procedure as a double bond.

Since Lewis never proposed any other bonding type in organic structural formulas, there is no other type of bond to address.

\section{The Even-Odd Rule}

The even-odd rule is a procedure to draw chemical structural formulas of molecules and ions. The structure is composed of one or several atoms of the periodic table.

As a reminder [9], the rule is as follows:

- Each atom:

- Is an element with one or several electron shells;

- Possesses an outer-shell filled with one to eight electrons;

- Has a number of electrons in the outer-shell, also called valence number, indicated in the periodic table;

- Has a valence number of the element giving the highest number of covalent bonds that the element can form.

- A structure meets the criteria below:

- When it is composed of only one atom, it forms no covalent bond;

- When it is constituted of several atoms, each atom forms a single covalent-bond with each of its first neighboring atoms. This covalent bond involves two electrons, one from each interconnected atom; 
- A covalent bond is represented by one line between both connected atoms;

- An atom may have zero, one or more than one line around it;

- In the 2D structure, each atom is represented by the letters from its element as in the periodic table;

- Two numbers have to be evaluated and written on each side of the atom.

- The left side number and the effective valence number:

- The left side number is the valence number as in the periodic table. It ranges from one for elements like sodium (Na) up to eight for noble gas like Argon (Ar);

- The effective valence number has to be evaluated: For a neutral atom, i.e. without charge, it is equal to the valence number; for a negatively charged atom, i.e. that possesses an extra-electron, it is the valence number increased by one; for a positively charged atom, it is the valence number decreased by one.

- The right side number of an atom:

- The right-side number, the "Lewis number", is equal to the sum of the effective valence number and the number of covalent bonds of the atom. It can also be expressed as the sum of the number of electrons left in the outer-shell and twice the number of covalent bonds.

- The Lewis number must be an even number. This is only possible when the number of bonds and the effective valence number are both odd or both even;

- The smallest value the Lewis number can take is zero: The atom has lost electrons from the outer-shell so it is empty and the atom has no bond;

- The Lewis number can range up to twice the effective valence number: this is twice the maximum number of covalent bonds for this element. This number is charge dependent through the effective valence number;

- If all atoms of a compound have Lewis numbers equal to eight, the compound is compatible with Lewis' octet rule.

- Electron pairs in the outer-shell of an interconnected atom:

- The number of electrons in the outer-shell is calculated by subtracting the effective valence-number and the number of covalent bonds. It is an even number;

- As a consequence, the outer-shell contains electron-pairs not involved in any covalent bond;

- This electron-pair number ranges from 0 to 4 whatever is the charge of the element;

- When this electron-pair number is 0 , no additional covalent bond can be formed by the element.

The even value of the right side number i.e. the Lewis number, and the even value of the number of electrons in the outer-shell are important keys to the validity of the even-odd rule. With these even values, molecules and ions belong to a group of electron-paired compounds [8] [9].

This rule and the modification of multiple-bonded structure will now be applied to redraw ions and molecules classically represented with multiple bonds between atoms.

\section{Application to Multiple-Bonded Compounds}

In the following, we purposely reduce the scope of the comparison to compounds containing elements of the main group of the periodic table. The proposed change of multiple-bonded compounds is illustrated and compared to classical structure. Table 1 lists neutral molecules, composed of charged or uncharged atoms, and Table 2 ions, also composed of charged or uncharged atoms. For each compound, the available classical structures and the modified even-odd structure are shown.

Classical structures of compounds, ions or molecules, are drawn first. The even-odd drawing follows with both associated numbers. In Table 2, classical structures of ions are specifically surrounded by square brackets.

When several classical drawings are available for the same compound, they are shown and specifically referenced.

In Table 1, 2D structural formulas of neutral molecules composed of charged and uncharged atoms are shown. The objective is to show that the overall neutrality of each molecule is conserved in both: classical structural formulas and the even-odd structural formulas.

The first example is the structure of nitrogen molecule, conventionally represented with a triple bond. With the even-odd rule, as proposed above, the triple bond is replaced by a single covalent-bond. The new structure is shown underneath with both numbers of the even-odd rule. The right side number is 6 , which means that this atom does not follow the octet rule. The difference between the effective valence number and the number of bonds 
Table 1. Comparison of structures drawn classically or following the even-odd rule: Application to neutral molecules. Molecules are ordered by increasing number of atoms. For each molecule, the classical representation is in the upper position. They can be easily identified thanks to the multiple bonds. Underneath, new designs are composed of only single bonds, no multiple bonds and two associated numbers: the left one is the valence number and the right one is calculated using the proposed even-odd rule.

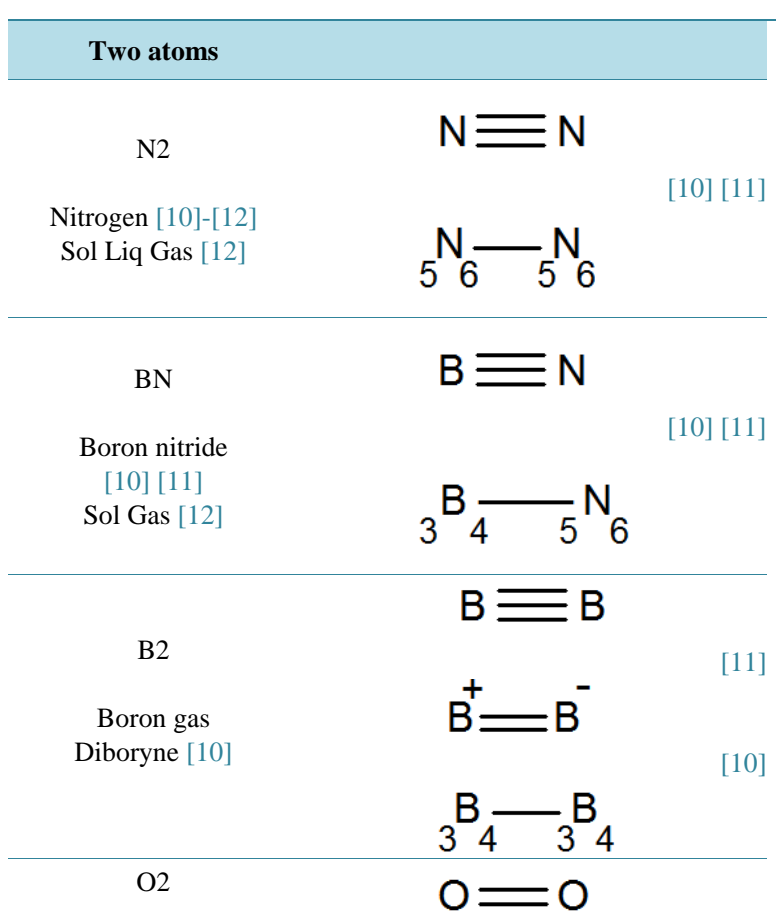

Oxygen [10]-[12]

Sol Liq Gas [12]

Different charge position

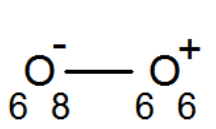

[10] [11]

\begin{tabular}{c} 
position \\
$\begin{array}{c}\text { Silicon carbide [10] } \\
\text { Sol Liq [12] } \\
\begin{array}{c}\text { Same charge position } \\
\mathrm{C} 2\end{array}\end{array}$ \\
\hline $\mathrm{C}=\mathrm{C}:$
\end{tabular}

Diatomic carbon

[12]

Dicarbon

[11] [12]

Stellar gas [12]

Same charge

position

[12]
Table 2. Comparison of structures drawn classically or following the even-odd rule: Application to ions. For each well-known ion, classical structures are drawn first, between square brackets. Multiple bonds are clearly identified. Underneath, new designs are composed of only single bonds between next neighbor atoms. Each element is associated with its valence number on the left side and an even right-side number calculated with the proposed even-odd rule. The charge position is clearly defined.

\section{Two atoms}

Cyanide anion $\quad[\mathrm{C} \equiv \mathrm{N}]^{-}$

[10]-[12]

Sol Liq Gas [12]

From hydrocyanic acid

${ }_{46}^{-}-\mathrm{N}_{5}$

$\mathrm{CN}(+)$

Monocyanogen [11]

Nitrilomethylium [10]

From cyanide

chloride

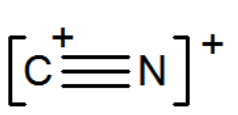

[10] [11]

$\mathrm{IO}(+/-)$

Iodosyl radical

[10] [11]

Gas [12]

from Hypoiodous

acid

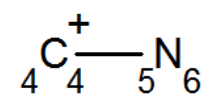

10] [11] [13]

$\mathrm{NO}(+)$

Nitrosonium

Ion [10]-[12]

In solution

$\mathrm{NO}(+/-)$

$[1 \stackrel{+/-}{=} 0]^{+/-}$

[10]-[12]

$$
7866 / 8
$$

$\left[\mathrm{N} \equiv \mathrm{O}^{+}\right]^{+}$

[10]-[12]

$$
{ }_{5}{ }_{6}-{ }_{6}{ }_{6}^{+}
$$

$[\mathrm{N} \equiv \mathrm{- \cdots}]^{+/-}$

Nitric oxide

[10]-[12]

From HNO

Nitroxyl [10] [12]

Or CINO

Nitrosyl Chloride

[12]
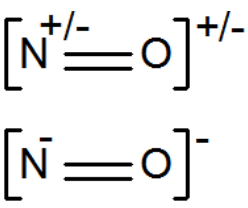

[10]

[11]

Boron oxide

[11]

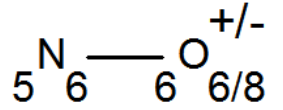

$[\mathrm{B}=\mathrm{O}]^{-}$

${ }_{3} \mathrm{~B}_{4}-\mathrm{O}_{8}^{-}$ 


\section{Continued}

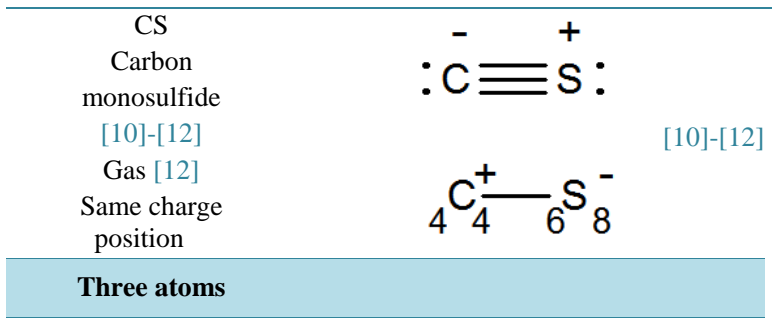

$\mathrm{HCN}$

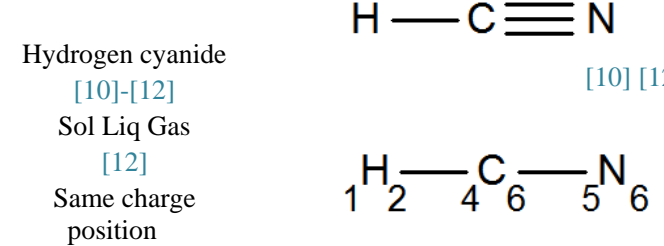

Cyanogen chloride

[10]-[12]

Sol Liq Gas

[12]

Same charge position

$\mathrm{N} 2 \mathrm{O}$

Nitrous oxide [10]-[12]

Sol Liq Gas [12]

Same and different charge position

$\mathrm{HNO} \quad \mathrm{H}-\mathrm{N}=\mathrm{O}$

Nitroxyl

[10]-[12]

Gas [12]

different charge posi-

tion

C3

$\therefore \mathrm{C}=\mathrm{C}=\mathrm{C}:$

Tricarbon [11] [12]

Stellar gas [12]

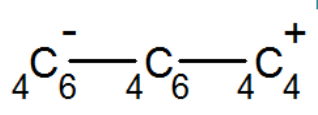

[11] [12]

$\mathrm{CaC} 2 \quad[\mathrm{C} \equiv \mathrm{C}:]^{2-}$

Calcium carbide

[10] [12]

Sol Liq Gas [12]

Different charge position
[10] [12]

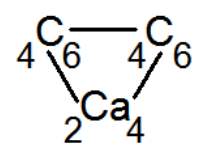

\section{Three atoms}

$\mathrm{CNO}(-)$<smiles>N#CO[Te]</smiles>

Cyanate ion

[11] [12]

NCO radical [11]

Oxomethylenamino

Radical [10]

In solution

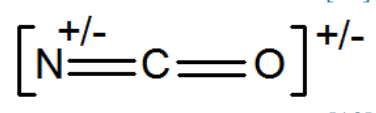

[10] [11]

$$
{ }_{5} \mathrm{~N}-\mathrm{C}-\mathrm{O}_{8}^{-}
$$

$\mathrm{CNO}(-)$

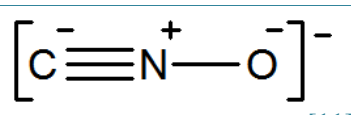

[11] [12]

Fulminate [11] [12]

$$
{ }_{4}^{-}-{ }_{5}^{-} \mathrm{N}_{6}^{+}-{ }_{6} \mathrm{O}_{8}^{-}
$$

$$
\left[\mathrm{H}-\mathrm{N}=\mathrm{N}^{+}\right]^{+}
$$

HN2(+)

Diazenyl radical [10]

Diazynium cation [10]

$$
[\mathrm{H}-\mathrm{N} \equiv \mathrm{N}]^{+}
$$

$$
{ }_{1} \mathrm{H}_{2}-\mathrm{N}_{6}^{+}-\mathrm{N}_{6}
$$

N3(-)

$$
\left[\mathrm{N}^{-} \stackrel{+}{\mathrm{N}}=\overline{\mathrm{N}}\right]^{-}
$$

Azide anion

[10]-[12]

$$
{ }_{5} \mathrm{~N}-{ }_{6} \mathrm{~N}-{ }_{8}^{-} \mathrm{N}_{6}
$$

OPO(-)or PO2(-)

$$
\left[\mathrm{O}=\mathrm{P}-\mathrm{O}^{-}\right]^{-}
$$

\section{Phosphinate \\ [10] [11]}

$$
{ }_{6} \mathrm{O}_{8}^{-}{ }_{5} \mathrm{P}_{6}^{+}-\mathrm{O}_{8}^{-}
$$

[10] [11]

$\mathrm{HCO}(-)$ or $(+)$

Formyl Radical

Solution [10]

$$
\left[\mathrm{H}-\mathrm{C}^{-}=\mathrm{O}\right]^{-}
$$

[10] [11] 


\section{Continued}

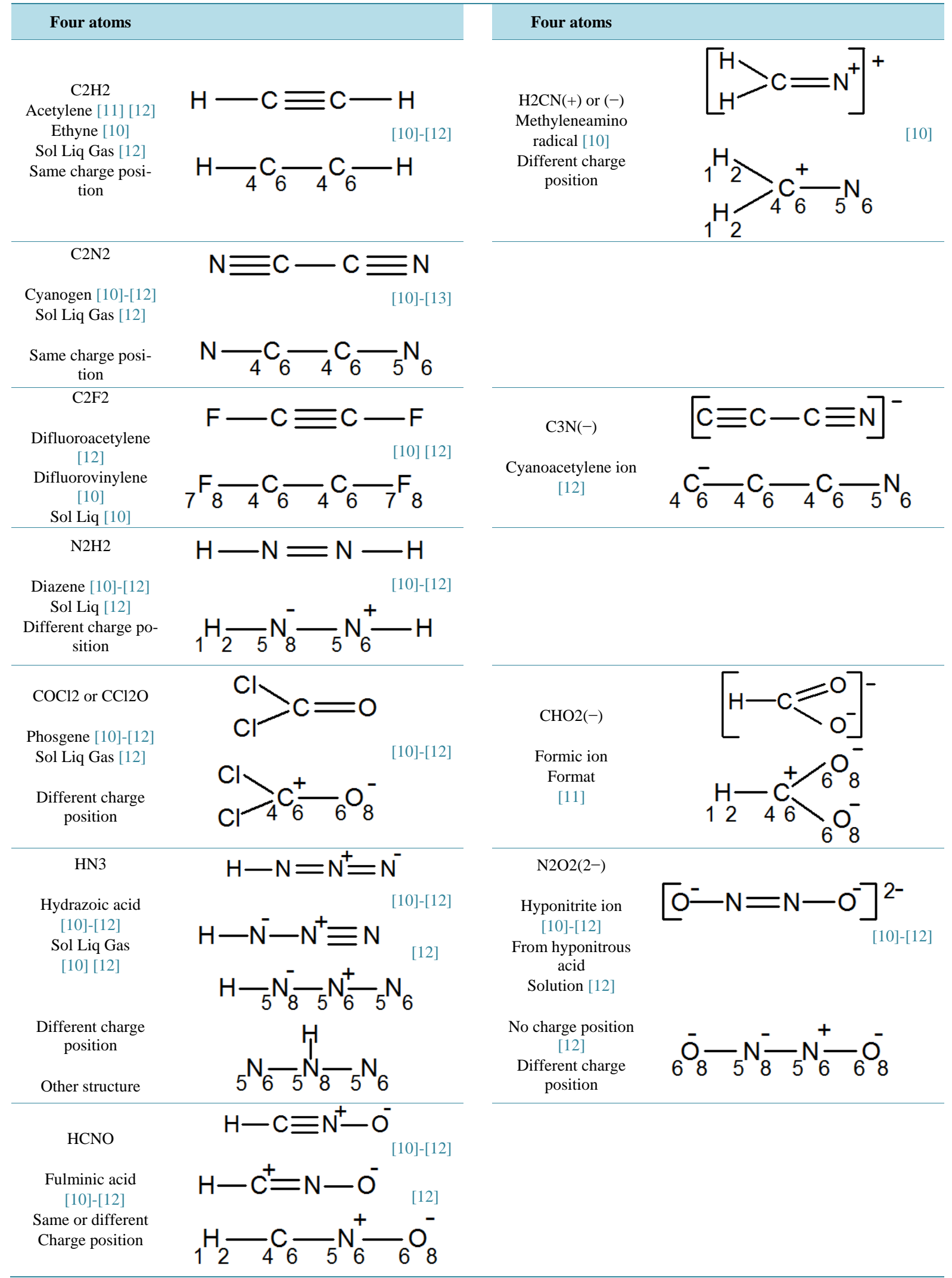




\section{Continued}

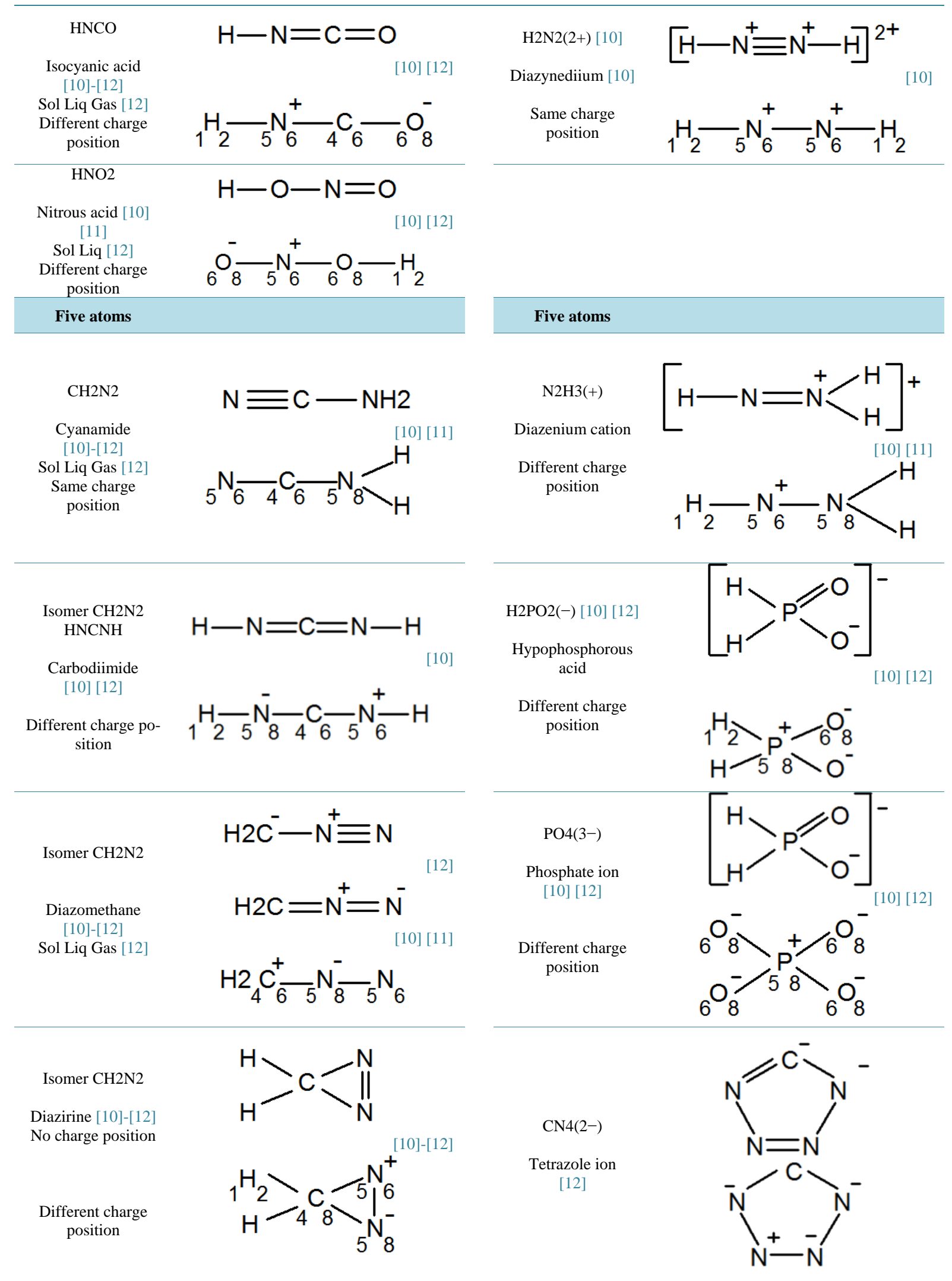




\section{Continued}

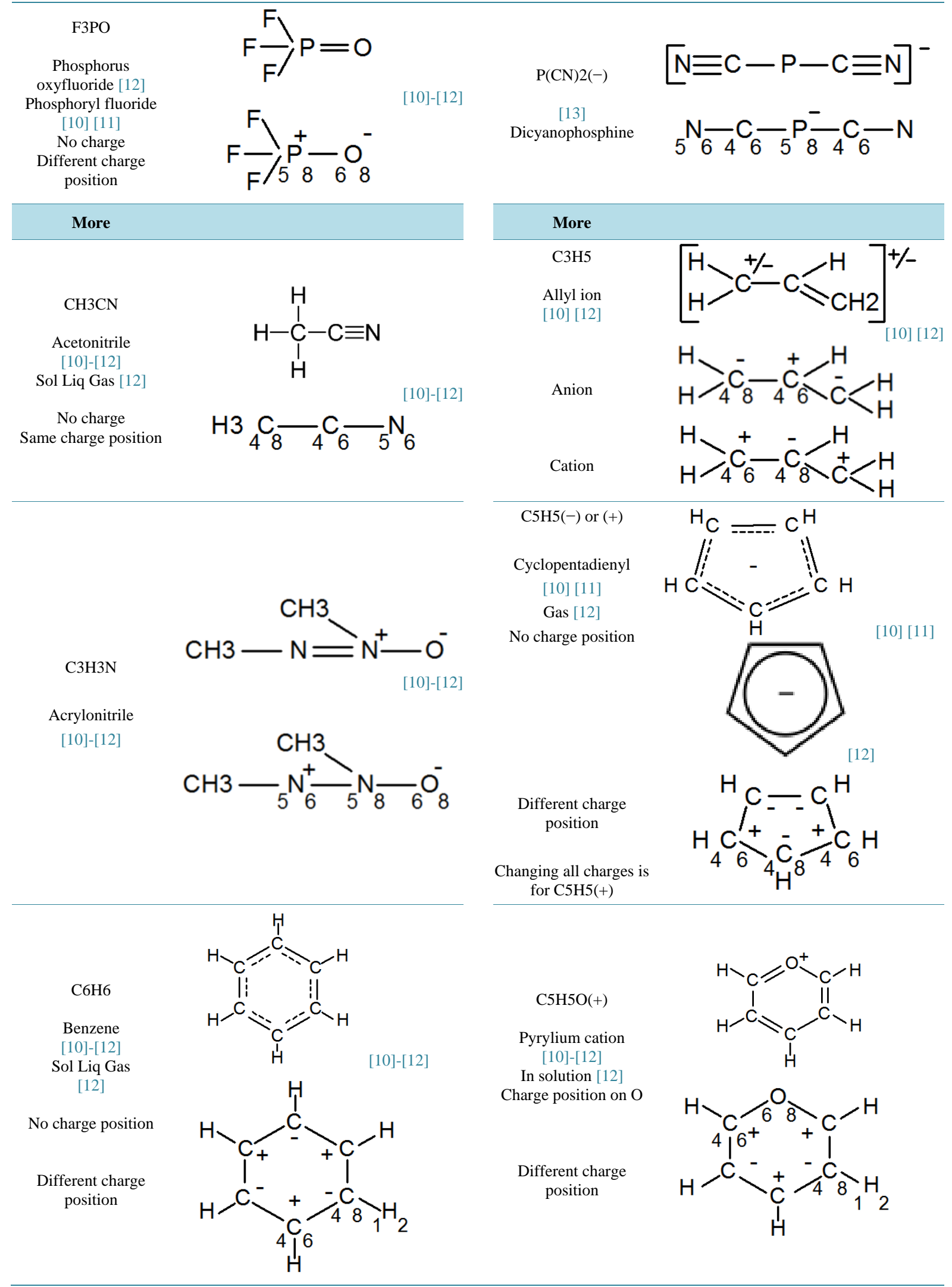




\section{Continued}

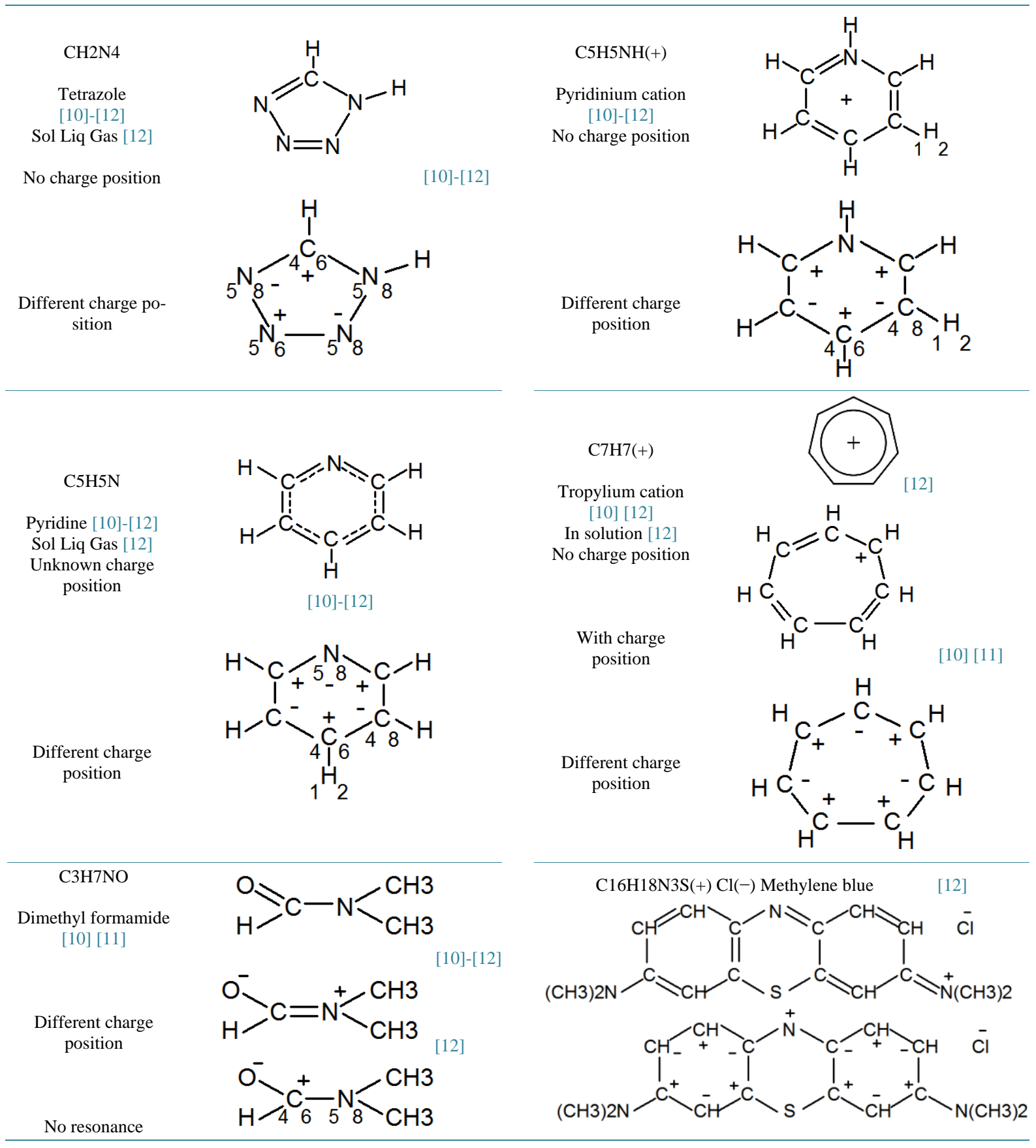

is equal to 4. This corresponds to the number of electrons in the outer-shell of the nitrogen molecule. The second structure in Table 1, uses the same structure but different elements. This molecule is resolved in the same operation.

The third molecule is di-boron. Two different classical structures are available. The first one uses a triple bond and the second one uses charges and a double bond. Both drawings end up as a unique even-odd structure after modification of multiple bonds. The left side number is 3 as in the periodic table for boron. The right side number is equal to 4 . The difference is equal to 2 and gives the number of electrons in the outer shell of the boron atom.

Still in Table 1, situated right below Boron, the di-oxygen molecule, classically represented with a double bond, is also drawn using the proposed modification rule. The resulting structure is composed of a single covalent-bond and two oxygen atoms bearing opposite charges. Both opposite charges cancel on a molecular scale to give a 
neutral total charge. Both atoms have a valence number of 6 , according to the periodic table. The negatively charged atom thus has an effective valence number of 7 , which, by adding the number of covalent bonds, gives a right-side number of 8 . The effective valence number of the positively charged atom is 5 , which gives a right-side number equal to 6 . Both values are even, in agreement with the even-odd rule. The difference between the effective valence number and the number of covalent bonds gives 6 electrons in the outer shell for the negatively charged oxygen and 4 electrons for the positively charged oxygen.

The same procedure is applied to all other neutral molecules in Table 1. It is of interest to note that in Table 1, the even-odd structure of PF3O is the only molecule following Lewis' octet rule.

Below in Table 1, one of the most complicated molecules is diazomethane (not diazirine): CH2N2. It is classically represented with two different structures, where charge locations depend on the position of multiple bonds. Applying the even-odd procedure on both classical structures result in only one possibility for charge positions and a unique design.

Far below in Table 1, cyclic molecules are addressed, like for instance the benzene C6H6 molecule. In classical structure, the charge is not precisely located. In the even-odd structure on the other hand, the charges are assigned to specific atoms and they cannot migrate.

On the whole, no difficulty was met in Table 1 to draw neutrally-charged multiple-bonded molecules.

In Table 2, chemical structural formulas of ions are listed. According to the proposed modification procedure, multiple covalent-bonds are replaced by single covalent-bond and assigned charges. The first ion has a triple bond which is replaced underneath by a single bond. This ion has one charge located on the carbon atom. According to the even-odd rule, the Nitrogen atom must be uncharged and the Carbon atom must be charged. The charge position for this ion is the same in both structures: classical or even-odd.

A little below, in both ions $\mathrm{IO}(+)$ and $\mathrm{IO}(-)$, denoted as $\mathrm{IO}(+/-)$, the charge is classically located on the iodine atom. The change from a double bond to a single bond implies shifting the charge from the iodine to the oxygen. In the new structure, the oxygen atom bears a charge, positive or negative. For this ion, the charge position is different according to the model used, classical or even-odd.

Two lines below, the nitric oxide ion $\mathrm{NO}(+/-)$ is represented with three classical structures and different positions for the charge. With the even-odd rule, this charge can only be assigned to the oxygen element, as explained in the following: The ion contains only one covalent bond and the valence number of the oxygen is 6 , which would give 7 on the right-side. To make it an even number, the effective valence number could be 6 or 8 , meaning that a positive or negative charge should be respectively assigned to the atom.

The same difficulty occurs below with the diazenyl ion HN2(+) in which the charge is assigned without ambiguity to the central atom by the even-odd rule.

In Table 2, this procedure is used for all other ions. On the whole, no difficulties were met to keep the same total charge and to reduce each classical multiple-bonded arrangement to a single-bonded one. Also in both Tables, the charge is always assigned without ambiguity to a specific element, resulting in outer-shells filled with electron-pairs as defined in the even-odd rule.

\section{Discussion}

\subsection{The Octet Rule in the Even-Odd Rule}

Historically, Lewis' octet rule greatly relies on multiple bonds. In Table 1 and Table 2, all organic compounds as Nitrogen, Oxygen, Hydrogen cyanide, Nitroxyl, Acetylene, Phosgene, Diazirine, Benzene, follow the octet rule in the classical representation. Once the even-odd rule and its criterion of single covalent bonds is applied, there are only very few of these molecules in which all atoms have an outer-shell filled with eight electrons: phosphorus oxyfluoride $\mathrm{F} 3 \mathrm{PO}$ and iodosyl radical $\mathrm{IO}(-)$.

Non-organic compounds were rarely compatible with Lewis' octet rule. The even-odd rule seems capable of addressing both types of compounds and unifies their representations.

\subsection{Kekulé and Non-Kekulé Compounds}

Kekule precisely described the classical benzene structure about fifty years before the octet rule. He proposed to represent it with alternatively multiple-bonded and single-bonded connections (see Table 1) [14]. This procedure was extended to other molecules [15]. Shortly after, molecules described as "non-Kekulé" were also represented 
[16] [17]. One of them appears in Table 2: tropylium cation, C7H7(+). This molecule is also drawn using the even-odd rule, i.e. without multiple bonds. As a consequence, the even-odd rule seems to encompass both types of Kekulé and non-Kekulé compounds.

\subsection{Two Successive Double-Bonded Connections for Neutral Atoms}

In tri-carbon, the center atom is classically represented with respectively two double covalent-bonds. While reducing each to single covalent-bonds and keeping an even right-side number, two charges must be assigned to the central atom. Two alternatives here: Either the same charge is assigned twice, resulting in the central atom bearing two charges that is electrostatically not very stable. Or two opposite charges are assigned resulting in a neutrally charged central carbon, thus electrically more stable. It seems possible that this procedure could be generalized but it has to be checked against several other ions and molecules. In both Tables, this pattern appears seven times.

\subsection{Comparison between $\mathrm{N} 2$ and 02}

It is common knowledge that $\mathrm{O} 2$ and $\mathrm{N} 2$ are chemically very different [12]. Elemental nitrogen, $\mathrm{N} 2$, found in the atmosphere, cannot be processed directly by living organisms whereas molecular di-oxygen, $\mathrm{O} 2$, is essential for cellular respiration in all aerobic organisms. This is not clear why because their classical structures are very similar: see Table 1. On the other hand, structures resulting from even-odd rule show a clear difference: Nitrogen is a neutral molecule with neutral atoms and the Oxygen has two active electrical charges. Differences in chemical properties could originate from charge location.

\subsection{Resonance/Mesomerism and Charge Delocalization in Electronic Structures}

Molecules classically represented using "resonance structures" also appear in Table 1 and Table 2.

In 1928, L. Pauling introduced the concept of "charge delocalization" in chemistry [18] [19], in order to explain why some structures drawn using Lewis' rule have a higher potential energy than observed in the real compound. Diazenyl ion $\mathrm{HN} 2(+)$ is for instance represented with two classical resonance structures: the charge can be either on the central nitrogen or on the outer one. By superposing both classical structures, both nitrogen bear half a charge. As an alternative, the even-odd structure assigns a charge to the central nitrogen. As a consequence, the resonance structure is not necessary for this ion.

A nearly identical pattern is proposed for dimethyl formamide; the last molecule in Table 1. Charges appear in one of the two classical structures. When applying the even-odd rule on the other hand, charges are clearly assigned to $\mathrm{C}(+)$ and $\mathrm{O}(-)$. No resonance structures are needed.

Another example: benzene, C6H6 molecule, in Table 1. Each carbon classically bears a charge of one half. With the even-odd rule, charges are assigned to atoms, alternatively positive and negative. They cannot shift from one atom to the next. Here as well, no contributing structure is necessary to represent the molecule.

The same can be written with all molecules classically represented using canonical forms in this paper. It would be of interest to apply the rule to other compounds, not listed in the present paper, in order to check if the same conclusion could be drawn.

\subsection{Symmetrical Structures}

In would seem natural that the charge position in a symmetrical ion should naturally respect this symmetry. This symmetry is unfortunately not preserved in many classical structures. In Allyl ion C3H5(+/-), azide anion N3(-) and methylene blue $\mathrm{C} 16 \mathrm{H} 18 \mathrm{~N} 3 \mathrm{~S}(+)$, central atoms do not bear a charge in their classical structures. The evenodd structures of these ions preserve their symmetrical characteristic in charge positions. This symmetry preservation property supports the coherence of the even-odd rule.

\subsection{Extension to Covalent Planar Layers in Crystals}

The structure of benzene shown in Table 1, is planar. By replacing the hydrogen atoms by carbon rings and keeping the planar form, an infinite carbon-ring layer is obtained: it is the structural formula of graphite [12]. The even-odd conservation rule can also be applied to this structure, resulting in only single bonds between carbons and alternating positive and negative charges. 
This planar layer structure, compatible with the even-odd rule, can also be found in alpha-boron-nitride crystals.

Another family of rhombohedral crystals has individual parallel layers following the even-odd rule; for iodides crystals, $\mathrm{MnI} 2, \mathrm{CaI} 2$ and $\mathrm{PbI} 2$; for bromides, $\mathrm{MgBr} 2, \mathrm{MnBr} 2, \mathrm{CdBr} 2, \mathrm{FeBr} 2$ and $\mathrm{CoBr} 2$; for chalcogenides, $\mathrm{ZrS} 2$ and alpa-TaS2 [12].

It seems thus plausible that the conversion of multiple bonds into single bonds is also applicable to classical crystal structures.

\section{Conclusion}

The hypothesis of the present paper is that all multiple covalent bonds in 2D structural formulas of ions and molecules could be replaced by single covalent-bonds. This modification causes internally a redistribution of charges. The scope of the even-odd rule, described in previous papers for well-known ions and molecules, is therefore expanded to multiple-bonded compounds and offers many advantages. This rule simplifies the drawing of compounds in several cases, for instance $\mathrm{HN} 2$ and $\mathrm{HN} 3(+)$ are represented here with only one structure. It preserves the symmetry of large compounds like methylene blue. Compounds like C6H6, classically represented with resonance structures, have now a fixed configuration. The distinctive chemical properties of $\mathrm{O} 2$ and $\mathrm{N} 2$ can be explained by the presence or the absence of local charges. This rule can also be applied to covalent planar solids like graphite. Finally, as the even-odd rule was applied to many known compounds in past and present papers, it confirms the ability of this rule to address covalent-bonded compounds such as molecules, ions or planar crystals.

\section{References}

[1] Couper, A.S. (1858) Sur une nouvelle théorie chimique. Annales de chimie et de physique, 53, 488-489.

[2] Loschmidt, J. (1861) Chemische Studien. Carl Gerold's Sohn, Vienna.

[3] Abegg, R. (1904) Die Valenz und das periodische System. Zeitschrift für anorganische Chemie, 39, 330-380. http://dx.doi.org/10.1002/zaac.19040390125

[4] Lewis, G.N. (1916) The Atom and the Molecule. Journal of the American Chemical Society, 38, 762-785. http://dx.doi.org/10.1021/ja02261a002

[5] Langmuir, I. (1919) The Arrangement of Electrons in Atoms and Molecules. Journal of the American Chemical Society, 41, 868-934. http://dx.doi.org/10.1021/ja02227a002

[6] Musher, J.I. (1969) The Chemistry of Hypervalent Molecules. Angewandte Chemie Internationale Edition, 8, 54-68. http://dx.doi.org/10.1002/anie.196900541

[7] Gillespie, R.J. and Popelier, P.L.A. (2001) Chemical Bonding and Molecular Geometry. Oxford University Press, Oxford.

[8] Auvert, G. (2014) Improvement of the Lewis-Abegg-Octet Rule Using an "Even-Odd" Rule in Chemical Structural Formulas: Application to Hypo and Hypervalences of Stable Uncharged Gaseous Single-Bonded Molecules with Main Group Elements. Open Journal of Physical Chemistry, 4, 60-66. http://dx.doi.org/10.4236/ojpc.2014.42009

[9] Auvert, G. (2014) Chemical Structural Formulas of Single-Bonded Ions Using the "Even-Odd" Rule Encompassing Lewis's Octet Rule: Application to Position of Single-Charge and Electron-Pairs in Hypo- and Hyper-Valent Ions with Main Group Elements. Open Journal of Physical Chemistry, 4, 67-72. http://dx.doi.org/10.4236/ojpc.2014.42010

[10] http://www.chemspider.com/

[11] http://www.ncbi.nlm.nih.gov

[12] http://en.wikipedia.org/

[13] Greenwood, N.N. and Earnshaw, A. (1998) Chemistry of the Elements. 2nd Edition, Butterworth-Heinemann.

[14] Kekulé, A. (1865) Sur la constitution des substances aromatiques. Bulletin de la Société Chimique de Paris, 3-2, 98-110.

[15] Wurtz, C.A. (1872) Sur un aldéhyde-alcool. Compte Rendu de l'Académie des sciences, 74, 1361.

[16] Dowd, P. (1972) Trimethylenemethane. Accounts of Chemical Research, 5-7, 242-248. http://dx.doi.org/10.1021/ar50055a003

[17] Tschitschibabin, A.E. (1907) Über einige phenylierte Derivate des p, p-Ditolyls. Berichte der Deutschen Chemischen Gesellschaft, 40-2, 1810-1819. http://dx.doi.org/10.1002/cber.19070400282

[18] Pauling, L. (1946) Resonance. Oregon State University Libraries Special Collections.

[19] Pauling, L. (1960) The Concept of Resonance. The Nature of the Chemical Bond-An Introduction to Modern Structural Chemistry. 3rd Edition, Cornell University Press, Ithaca, 10-13. 
Scientific Research Publishing (SCIRP) is one of the largest Open Access journal publishers. It is currently publishing more than 200 open access, online, peer-reviewed journals covering a wide range of academic disciplines. SCIRP serves the worldwide academic communities and contributes to the progress and application of science with its publication.

Other selected journals from SCIRP are listed as below. Submit your manuscript to us via either submit@scirp.org or Online Submission Portal.
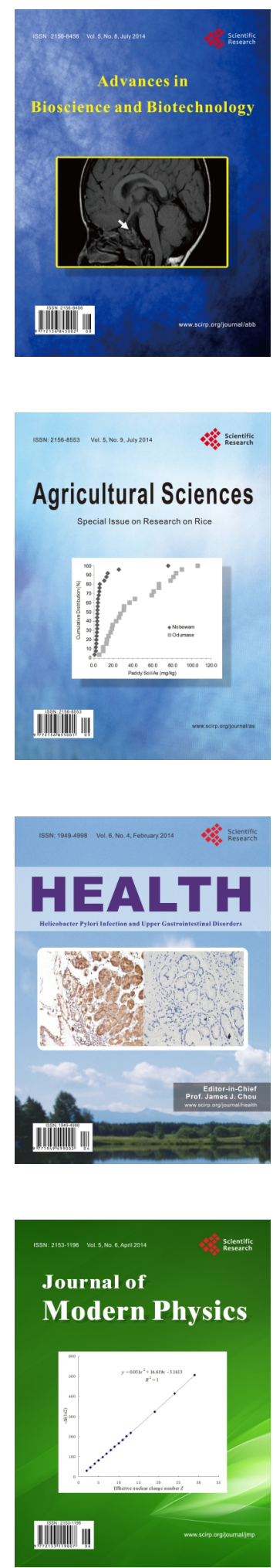
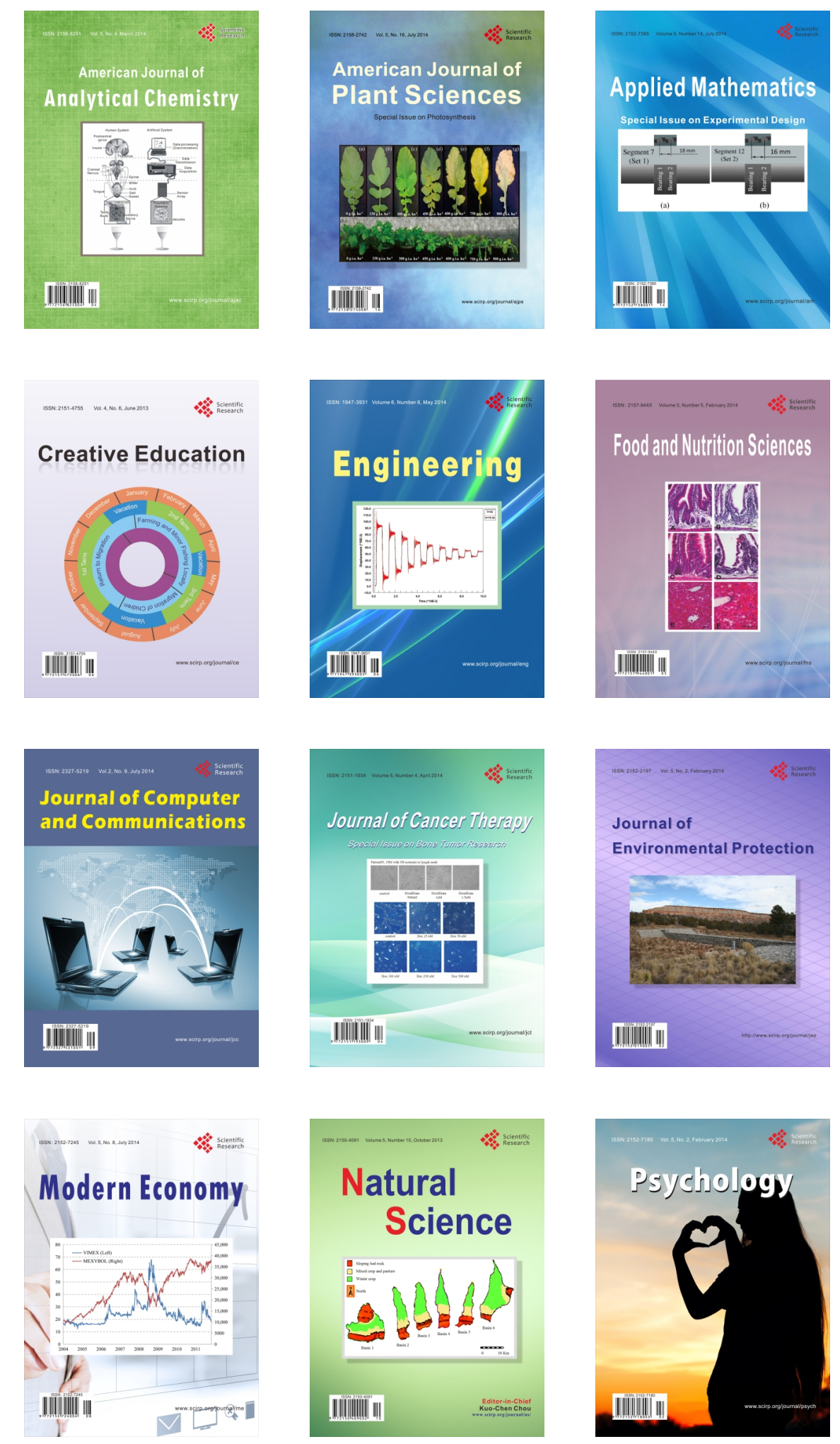